\title{
Behavioral and neural correlates of visual preference decision
}

\section{Shinsuke Shimojo}

Shinsuke Shimojo, "Behavioral and neural correlates of visual preference decision," Proc. SPIE 7240, Human Vision and Electronic Imaging XIV, 724003 (10 February 2009); doi: 10.1117/12.817151

SPIE Event: IS\&T/SPIE Electronic Imaging, 2009, San Jose, California, United States 


\title{
Behavioral and neural correlates of visual preference decision
}

\author{
Shinsuke Shimojo \\ 1) California Institute of Technology 139-74, Division of Biology, \\ Computation and Neural Systems, Pasadena, CA 91125, USA. \\ 2) Japan Science and Technology Agency, ERATO Shimojo, Implicit Brain Function Project, NTT CS \\ laboratories, Atsugi, Kanagawa, 243-0198 Japan.
}

\begin{abstract}
Three sets of findings are reported here, all related to behavioral and neural correlates of preference decision. First, when one is engaged in a preference decision task with free observation, one's gaze is biased towards the to-be-chosen stimulus (eg. face) long before (s)he is consciously aware of the decision ("gaze cascade effect"). Second, an fMRI study suggested that implicit activity in a subcortical structure (the Nucleus Accumbens) precedes cognitive and conscious decision of preference. Finally, both novelty and familiarity causally contribute to attractiveness, but differently across object categories (such as faces and natural scenes). Taken together, these results point to dynamical and implicit processes both in short- and long-term, towards conscious preference decision. Finally, some discussion will be given on aesthetic decision (i.e. "beauty").
\end{abstract}

Key words: preference, decision making, gaze, memory, face

\section{INTRODUCTION - Gaze, orienting and preference}

Eye gaze has some distinctive functions in a variety of species, even among monkeys and great apes in the wilderness.Gaze and gaze shifts are also critical elements in the newborn's sensory-motor repertoire. "Preferential looking", or selective gaze, can be easily observed from several-hour to year-old infants (including monkey infants as well). It serves as a basis for sensory-motor coordination on the one hand and social-cognitive development on the other hand (Haith, et al., 1977).

Gaze shifting is just one example of bodily orienting mechanism. Other examples may include head turn, body turn, turning of toes, and weight shift. Such orienting mechanism may be one of the earliest sensory-motor functions ontogenetically, as mentioned above. It may also be found in most primitive species, thus considered very old evolutionarily.

Considering such a long phylogenetic history and functional significance, one may speculate that gaze is still the basis of and an indispensable condition for sensory-motor coordination and attention as well as memory and learning in the human adult.Here, we will step even further arguing that gaze orienting is an implicit somatic precursor of even higher-cognitive functions such as preference and decision making.

\section{THE GAZE CASCADE EFFECT}

\section{2-1. Gaze bias preceding preference}

To address these issues, we performed a $2 \mathrm{AFC}$ (two-alternative forced-choice) experiment in which the subject was asked to observe two faces presented on a CRT monitor to make a preference judgment (Shimojo,et al.,2003). There 
was no fixed time limit, and the subject can freely move his or her eyes to compare the two faces. The subject was asked to press one of two buttons to indicate his or her preference as quickly as possible. The faces were kept on the screen until the subject made a response. Base-line attractiveness within pair was matched to remove any potential effects on preference. Eye movements were recorded with an eye tracker (EyeLink2)to examine whether gaze could predict preference choices.

The data weredivided into $200 \mathrm{~ms}$ time blocks and the likelihood of the gaze towards the to-be-chosen face was calculatedfor each block. When we time-locked the eye-movement data to the stimulus onset and averaged across trials, we did not find any significant bias, other than the strong general tendency of the initial gaze towards left. A very intriguing gaze pattern was found only when we time-locked the eye-movement data.

The likelihood of the gaze was roughly .50as expected until approximately $1000 \mathrm{~ms}$ prior to subject response, when the probability jumped past .80 (red curve in Fig. 1, which shows the best-interpolated sigmoid curve).

When the subjects performed other tasks, such as choosing the rounder of two faces(roundness task; blue curve in the figure) or the least attractive face (dislike task; gray curve), the gaze likelihood curves elevated somewhat, but level off at 55-65\% level, in contrast to the preference task (the other two curves in Fig. 1). Note that task difficulty did not differ significantly between studies, as estimated from the means of performance time (ibid). Therefore,such a plateau-like elevation in the control task may be interpreted as reflecting preparation for the response (i.e., the subject tended to look fixate on the chosen face before choosing it). The extremity and the distinctive pattern of elevation in the preference task (as indicated by the vertical red arrow in the figure), on the other hand, suggest that the gaze cascade is unique to relative attractiveness.

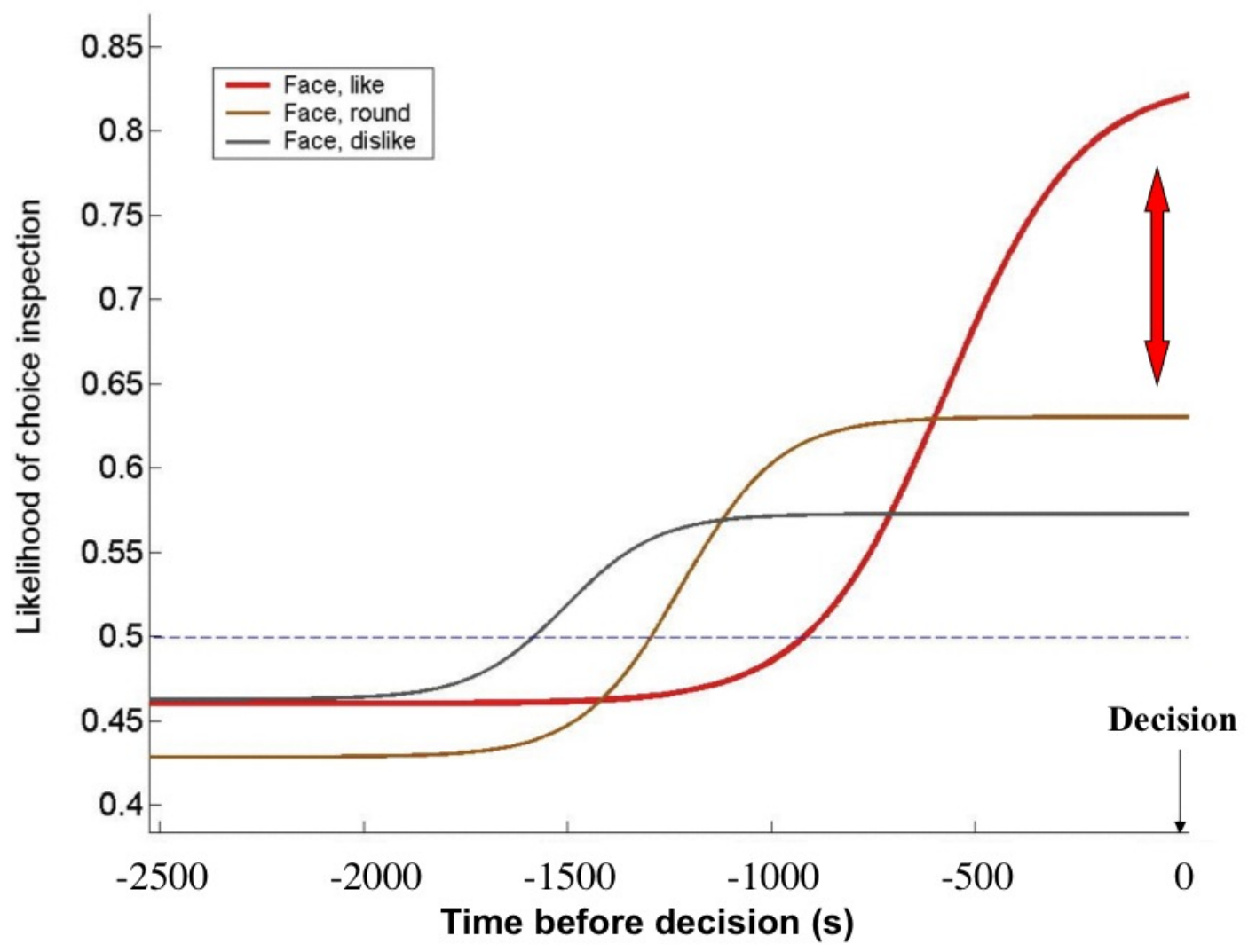

Figure 1. Gaze cascade effect in preference choice task.

The gaze cascade effect,which had turned out to be a distinctively unique characteristic of the preference decision process, raised animportant question. How general and robust is the gaze cascade effect, across various conditions and stimuli? Just to show one example from our long series of experiment we repeated the experiment while 
maximizing the average difference in attractiveness within pairs (Shimojo, et al., in press). As a result, we obtained a very similar gaze cascade curve. The amplitude of elevation (to $74 \%$ ) was significantly weaker than (83\%) in the original experiment with minimum attractiveness differences, yet the other parameters to fit a sigmoid function (such as the onset timing) were highly similar.

\section{2-2.Is the gaze bias a critical part of the decision-making process of preference?}

The second main issue that was raised by the initial finding was whether orienting mechanisms (such as that for gaze shift) underlies, precedes, and plays an indispensable role inthe preference decision-making process. If orienting mechanisms turn out to be a necessary component (at least under a natural free-view condition), it would be a very intriguing finding, particularly from the evolutionary and the developmental viewpoints. There are at least two lines of evidence to believe so.

In what we call the "peep-hole experiment," we presented two faces side by side on the display as before (Simion\& Shimojo, 2006a). The task was the same 2AFC preference decision. This time, however, the faces are mostly occluded, except for a small window that moves contingently with the gaze (Fig. 2). The size of the visibility widow was adjusted such that only one facial feature could be seen at a time. Needless to say, this makes the task more laborious, and the subjects inevitably made large numbers of saccades within and across faces (the average performance time extended up to $29-31 \mathrm{sec}$, from 3.1-3.6 sec in the original experiment).

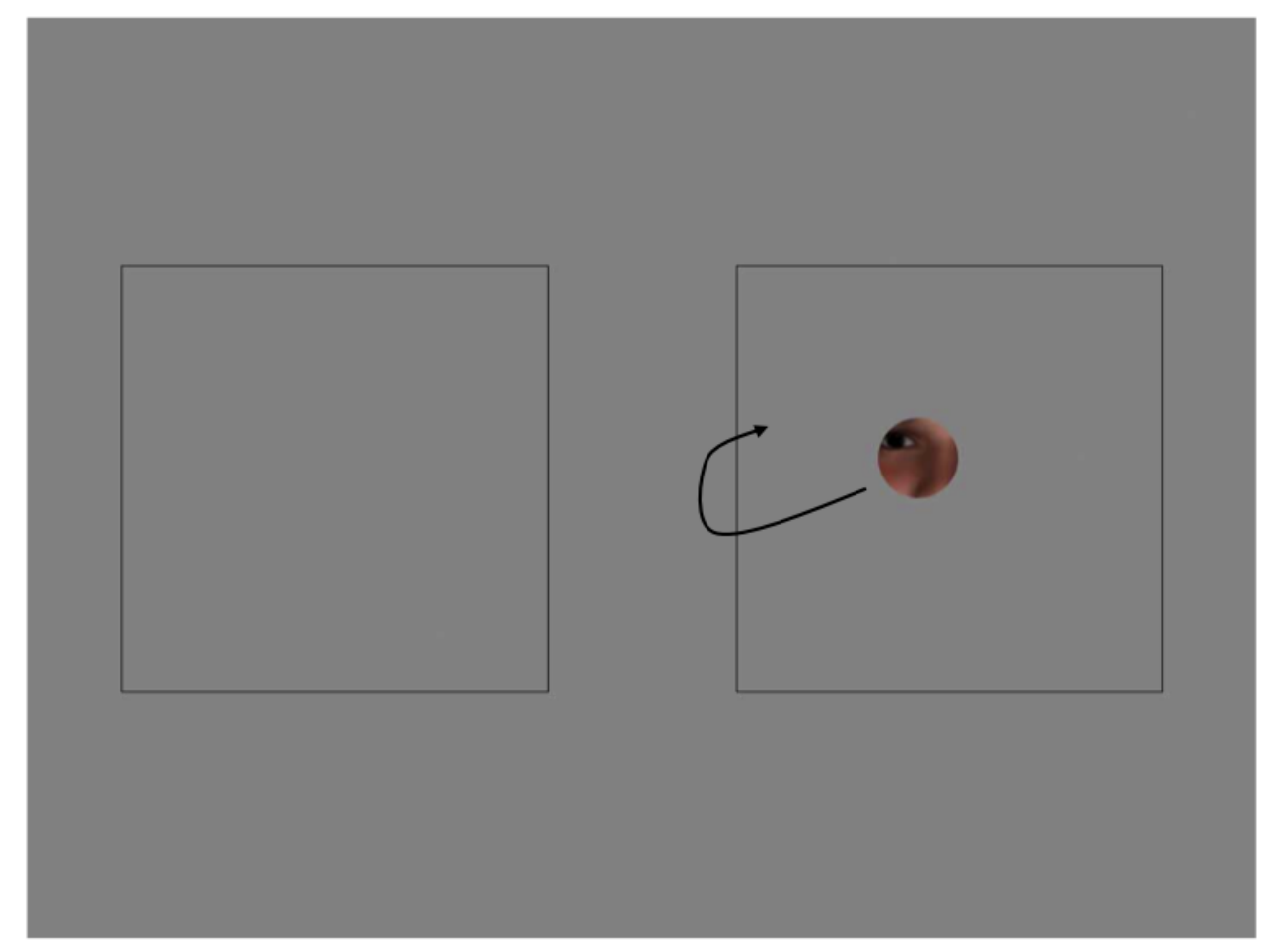

\section{Figure 2. Peep-hole experiment - Stimulus display (a snapshot).}

As a result, the onset time of the bias (according to the best fit with a sigmoid function) was $7.5 \mathrm{sec}$, as opposed to $0.8 \mathrm{sec}$ in the original experiment. This finding has an indirect but significant implication as to our main question(i.e., whether the orienting mechanism is intrinsically involved in the decision making process of preference). More than several seconds before the final response, the subjects were still vigorously collecting local sensory information (perhaps 
to construct internal representations of the two faces which are detailed enough for preference decision). However, such local sensory sampling was already starting to bias towards the final choice.

In another experiment, we addressed the following question:if the gaze shift is an intrinsic component of the process towards preference decision making, then we may be able to manipulate preference decisionsto some extent by manipulating their gaze behavior. This would be even stronger evidence for gaze as an intrinsic component of the preference decision making process.

We again presented two faces side by side, but unlike our original paradigm, we presented one face and the other alternately with different durations $(900 \mathrm{~ms}$ vs. $300 \mathrm{~ms}$; Shimojo et al., 2003). This cycle of presentation was repeated twice, 6 times, or 12 times. Note that the same face was presented always on the same side (right or left) of the screen, but never presented simultaneously. Towards the end of each trial, the two faces are briefly presented simultaneously (500ms), and the subject had to indicate which face is more preferable by pressing a button.

The preference was at chance level between the longer- and the shorter-duration faces after 2 cycles of repetition, but significantly above chance level after 6 and 12 cycles $(59.0 \%, p<0.001$ for cycle, and $59.2 \%, p<0.005$ for 12 cycle, respectively). A qualitatively same result was obtained when the faces were presented top and bottom of the screen $(60.2 \%, p<0.0001)$; thus the manipulation effect is not related to the specific layout of the faces.

Our control experiments in which the subject was not allowed for free inspection with gaze shift, the bias disappeared. Moreover, such an effect by gaze manipulation on choice was not observed when the subject was engaged in the face roundness task. Thus, gaze is intrinsically related only to the preference choice task, and spontaneous gaze shift, as opposed to passive exposure, is necessary for such manipulation effect to occur.

To consistently interpret both the gaze cascade findings and this set of manipulation findings, the "gaze as an intrinsic component" seems to be the only simple and feasible account.

It may also worth mentioningthatinterviewing subjects after the session, the majority of the subjects did not notice the bias in presentation durations (and thus their gaze bias), and even those who did noticedenied any causal relationship of it to their final preference decision.

One may still ask how strong this manipulation could be. To address this, we prepared a series of face pairs, whose baseline differences in attractiveness had systematically varied from minimal to maximal (Simion\& Shimojo, 2006b). The expectation was that perhaps minimal differences in attractiveness might be easily reversed by gaze manipulation, but maximal difference may be more difficult. Against this expectation, our resultssuggests that even the largest baseline differences can be partly reversed by the gaze manipulation, approximately at the same frequency $(15 \%$ of trials) as minimal differences. Thus, the gaze manipulation turned out to have a substantial influence on the subject's preference decision.

\section{2-3. Neural correlates of preference decision}

To identify the neural correlates of processes (including the gaze bias) that precedes the conscious preference decision, we recorded EEG (32 channels) while the subject performed the preference tasks (on faces or Fourier descriptors), control tasks (roundness, or dislike of face), or passively viewing the stimuli (Bhattacharya, Simion,\& Shimojo, unpublished). The results indicated that there was stronger neural activity in the frontal channels in the preference tasks regardless of the stimuli.

Encouraged by these EEGdata, we next performed an fMRI study to localize the brain regions that are relevant to the preference decision task, fMRI may be more preferable. There are, however, a couple of factors that made the original gaze cascade paradigm unsuitable to obtain meaningfulfMRI data. First, presenting two faces prevents us from contrasting brain response to one face (say, chosen as more attractive) against that to the other. Second, allowing free inspection, thus eye movements, add extra noise to the MRI signals. For these reasons, we abandoned the original paradigm, and devised another behavioral paradigm of preference decision which was in our opinion much more suitable for fMRI (Kim,Adolphs, O'Doherty, \& Shimojo, 2007).

While it was still a $2 \mathrm{AFC}$ preference decision task, only one face was presented briefly $(50 \mathrm{~ms})$ at a time, then the other, sequentially with a random variable interval $(1-3 \mathrm{sec}$.). The same two faces were thus repeatedly presented sequentially until the subject made a choice. Note that this procedure allow us to isolate the brain responses to each face, and to search for neural signals that predict the conscious preference decision later. Also, it was expected to enable us to overcome the limitation in the temporal resolution of fMRI, and to identify dynamic changes of activity in the brain, from the initial stimulus presentation to the final response. Thus we call it the TED (Temporally Extended Decision making) paradigm. Owing to this paradigm, we expected to see an earlier contrast (e.g., more neural activation to the tobe-chosen face) at a sub-cortical level, and a later contrast at a cortical level. 
Since most of the subjects responded during the second cycle in the vast majority of the trials, our analysis concentrated on the second-cycle trials. In the results, there were three distinctive areas that were activated during the preference decision between faces: the nucleus accumbens (NAC), the medial orbito-frontal cortex (mOFC), and the operculum/insula (OP/INS).

The dynamic changes of neural activity in these areas were of particular interest. As reported in the original paper (Kim, et al., 2007), the three areas had different peak timings: the NAC showed a very early difference in the first cycle, while the mOFC had its activity peak in the second cycle. Interestingly, activity in these areas decreased towards the response, and instead, the OP/INS increased their activity presumably to prepare for the motor response. The overall pattern of the results was highly consistent with our expectation in that a) there were differential signals in sub-cortical regions that predict the conscious preference decision, but b) it was replaced by differential signals in cortical areas before the motor response.

Since the NAC is a sub-cortical area known to be activated during reward-related learning, preference decision and affective aspects, it may be reasonable to assume that activation of this area may be the implicit precursor of conscious preference decision. Additional experiment of ours addressed this issue, and indeed obtained confirmative evidence (ibid). Fig. 3 compares the fMRI result when the subject was engaged in a face roundness task while the fMRI signals were contrasted with regard to their preferences (which was behaviorally taken in the subsequent session) on the left, and the result during the explicit face preference task on the right. As obvious from the figure, the same NAC are was activated with regard to preference in the early cycle, regardless of the explicit task that the subjects were engaged in.

\section{Implicit Preference Decision from Group Explicit Preference Decision from Group 2 (Early cycle during Roundness decision) 1 (Early cycle during Preference decision)}
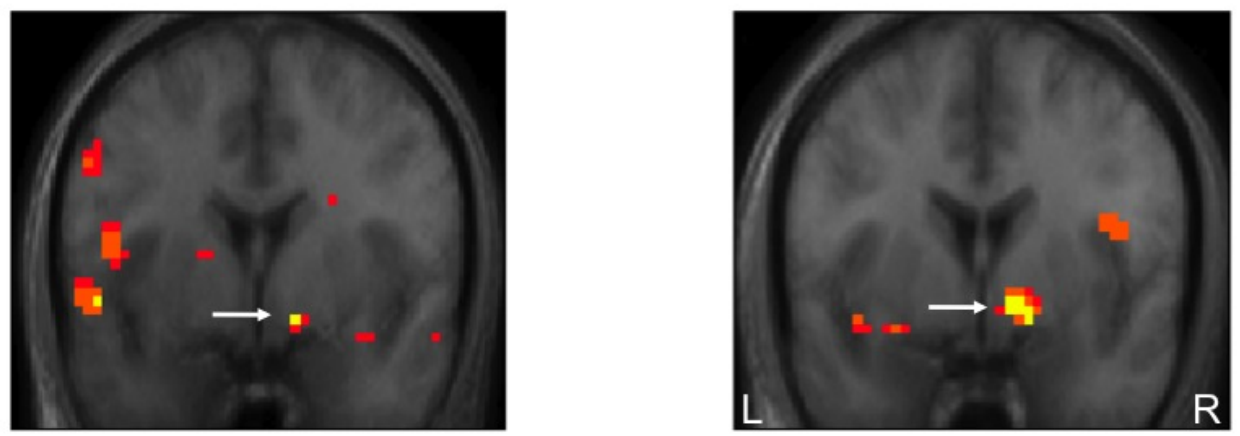

\section{Figure 3. fMRI results in the implicit (i.e. roundness; left) and the explicit (right) preference choice tasks. Both are during the early cycle (i.e. the first look).}




\section{2-4. Beauty judgment?}

A question that had been asked frequently was whether or not the gaze cascade occurs when the task is not on attractiveness, nor objective feature (such as roundness of face), but rather directly aesthetic - i.e. which of the two stimuli is "more beautiful."

Beauty judgment may be considered somewhere inbetween the attractiveness decision which is considered most subjective, and objective control tasks. The attractiveness decision is considered subjective because there is no publically "correct" answer, and the decision is entirely based upon criteria and factors internal to the observer. The control tasks such as roundness of face are objective because there is always an objectively correct answer due to some physical criteria. Relative beauty is trickier with this regard, because one may argue that there are indeed objective criteria of beauty (in each object category such as face), but even those are both culture- and individual-dependent, as well as context-dependent. A prediction from this consideration would be that indeed the gaze cascade would occur in the relative beauty task, yet its magnitude would be only intermediate.

Yet another view on the relationship among these decisions would be the following - When one is asked on (relative) beauty, it is almost equivalent to be asked to mentalize others' attractiveness decision, i.e. "which one of the pair the vast majority of other observers choose as more attractive (not asking which you would choose as such)?" This may invite partly different cognitive/neural mechanisms such as that of "TOM (Theory of Mind)," the gaze pattern before the conscious decision may be qualitatively different from those observed in the attractiveness or the objective decision tasks.

Whereas we do not have a full set of data to answer this question, we do have some preliminary data (unpublished) indicating that the gaze cascade may occur even in relative beauty choice, and its amplitude could be as strong as in the attractiveness choice.

\section{2-5. Gaze cascade effect - summary}

As explained in the introduction, these findings are consistent with implicit orienting mechanisms as the developmental origin of conscious emotional decision. Implicit bodily preference precedes explicit conscious preference, not vice versa, both in the developmental sense as well as in the "on-line" process leading to preference decision in adults.

Thus all together, our "gaze cascade" findings point strongly to an enactive view on preference decision making. That is, orienting action and cognitive decision are tightly linked together in a positive feedback system which spreads over time. Whereas qualitatively the same gaze cascade occurs in preference task on non-face stimuli such as geometric figures (Shimojo et al., 2003), it may contribute to a social coevolution scenario between people's sensitivity to other's gaze on one hand, and capability of generating and expressing liking by gaze on the other hand.

\section{MEMORY EFFECTS ON PREFERENCE}

\section{3-1. Familiarity vs. Novelty principles}

As demonstrated in the gaze cascade effect, understanding preference decision making is a challenging problem mainly because the underlying process is partly implicit and dynamically evolving over time. Such a dynamical process is meant to be an interaction between orienting and visual mechanisms over several seconds in the case of the gaze cascade effect, but one may also consider longer-term effects which are dependent on context and past experience.

What is really intriguing, and peculiar at the same time, is that there is evidence for both familiarity and novelty as a critical factor for preference decision, in adults and infants. The well-known "mere exposure effect" (Zajonc, 1968) should be considered as evidence for the familiarity principle, whereas novelty preference has also been reported repeatedly in many species, and particularly in specific stimuli such as natural scenes in the human. To resolve this seeming contradiction, we examined cumulative effects of exposure in different categories of object (Shimojo, et al, 2007).

Faces, geometric figures, and natural scenes were tested separately in 3 blocks with the order counterbalanced across participants. Each block has 8 sets of trials, in each of which 26 pairs of within-category images were presented sequentially, and for each pair the participant judged relative preference in a 7-point scale. In each set of trials, one image was repeatedly presented at randomized location ( $\mathrm{L}$ or $\mathrm{R}$ ), paired with a new image each time. Thus except for the first trial, the pair/choice was always old vs. new.

When probability of preference for the old stimulus was plotted against trial number (Fig. 4), faces showed a steady increase, thus a general tendency towards familiarity preference. There is no tendency of novelty preference throughout the face set. On the other hand, natural scenes showed a quick decrease with habituation, and a tendency 
toward novelty in general. Geometric figures stayed neutral over trials, thus no strong bias either way. The overall pattern of results points to a possibility that Familiarity and Novelty principles are segregated across object categories. Different social/ecological significances may partly account for it.

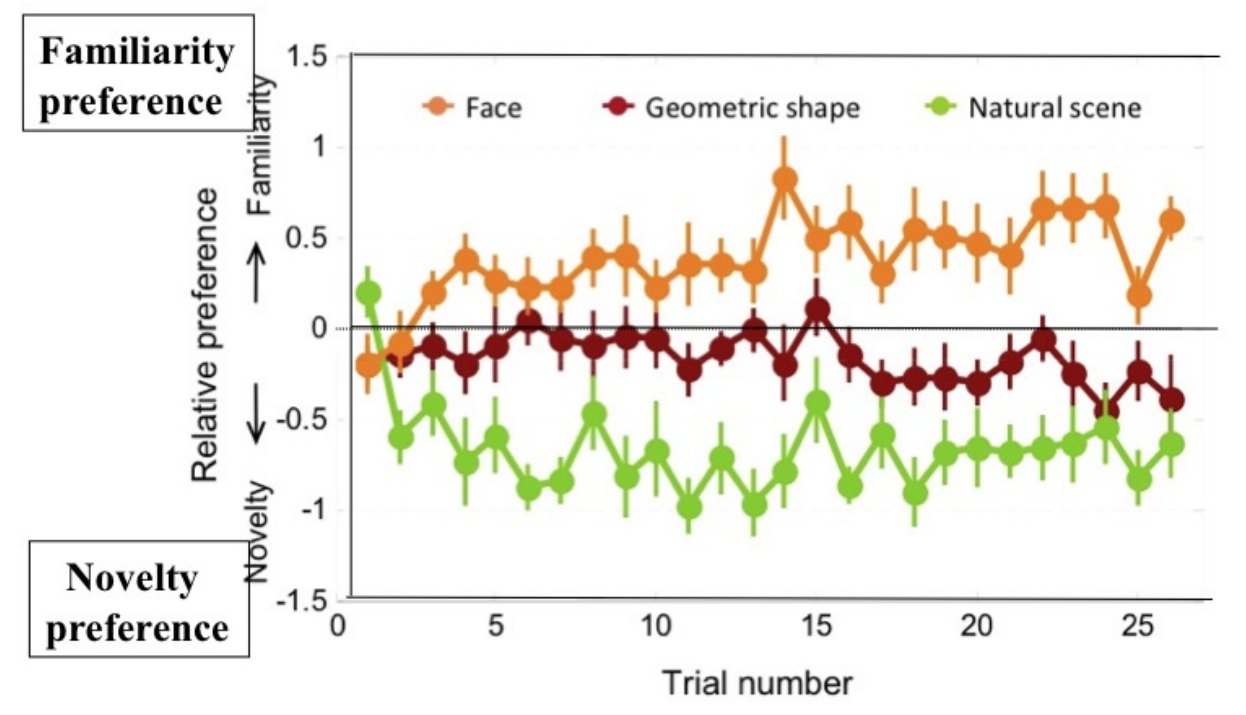

\section{Figure 4. Relative preference as a function of experience.}

\section{3-2. Intrinsic differences among object categories, or is it a matter of plasticity?}

While the segregation may indicate intrinsic biological differences among object categories, it may also be due to differences in similarity/variability within category between faces and NS. For example, one may hypothesize that the brain determines attractiveness of visual stimulus due to only two grand criteria. One is that the more novel a particular stimulus is, the more brain learns, thus more attractive. The other is that the more frequently the brain encounters the same or similar stimuli then learning is more useful, thus more attractive.

These two criteria may nicely correspond to Novelty and Familiarity principles, and potentially account for the segregation of these principles between faces and natural scenes. This is because faces are in general very similar to each other in terms of physical features, and over-trained ever since childhood. On the other hand, natural scenes vary a lot across lower-level visual attributes, such as luminance and color, spatial frequency, orientation, etc. Thus, mere distance in such a feature space may matter more.

To examine this issue, we ran two additional experiments (Shimojo, et al, 2008). First, we reused the same faces and natural scene stimuli but presented them upside down in the same 2AFC preference task, to see if the critical factor is orientation-specific and holistic. The results faithfully duplicated the pattern of the original results, i.e. Familiarity in faces and novelty in natural scenes. This may indicate that orientation-nonspecific, lower-level local features may be critical.

Second, we directly manipulated variability within object category; i.e. to introduce more variability within faces by including cartoons, and natural face-like patterns, etc., and more similarity within natural scenes. As a result, the 
familiarity preference did not significantly decreasein faces even with more variability. In natural scenes which are more similar to each other, however, the novelty preference were substantially weakened. In addition, we ran two sets of geometric figures; one with high similarity within, and the other with low similarity. We found an increasing bias towards familiarity preference in the high similarity set, but no such bias in the low.

The overall pattern of results is consistent with a notion that increasing similarity in local features shifts preference from novel to familiar stimuli, in close relation to the average hypothesis of attractiveness and the efficient coding theory of perception. However, The robust persistence of familiarity preference in faces with more variance is a distinctive exception that requires more investigation.

One possibility is that indeed face is a biologically and socially special category that requires entirely different criteria of preference as well as neural mechanisms. Another feasible account is that as such, faces are over-trained with lots of reward values ever since early childhood and thus "imprinted" in the critical period. That may also explain the resistance of the face category to a short-term manipulation of variance in the laboratory.

\section{3-2. Dependence on task?}

Yet another issue raised by the initial set of "segregation" findings would be that the findings are partly inconsistent with the "mere exposure" effect (Zajonc, 1968), i.e., repeated exposure to a visual object, regardless of the object category, leads to an increase in preference. The inconsistency may be partly due to what exact task was repeatedly performed during the experience phase: preference decision, choice of any kind, or mere exposure. To address this issue, we conducted the same two-alternative force-choice preference judgment as Shimojo et al. (2007)(Liao \& Shimojo, 2008 ). In the current experiment, the same old stimulus was again presented always with a new stimulus, following either of the two kinds of experience phases: passively viewing all the paired pictures, or performing an objective judgment on the paired stimuli (on roundness of face, color temperature of natural scene, or complexity of geometric figure).

The results showed that after passive viewing the old stimulus was preferred significantly more frequently in faces, thus a mere exposure effect occurred, but not in natural scenes or geometric figures. Afterrepeated objectivejudgment in the experience phase, the novel stimulus was preferred significantly more frequently in both geometric figures and natural scenes. It possibly indicates a habituation at the selection level for certain object categories.

The overall results further confirm the segregation of novelty and familiarity principles across object categories, and indicate uniqueness of face as a special kind of stimulus. They also suggest that different tasks lead to memory effects at different levels such as perception and selection.

\section{GENERAL DISCUSSION AND CONCLUSIONS}

We examined two aspects which are both critical to preference decision. One was eye-orienting as a "somatic precursor" of conscious decision (the gaze cascade effect). The other was effects of memory on preference decision: Familiarity preference in faces, and novelty preference in natural scenes, in particular. One would ask how these are intrinsically related in a more grand framework of neural information processing, from sensation to decision, and to action.

The gaze cascade findings clearly indicated that implicit orienting mechanisms precede and lead to conscious preference decision. However, it immediately raises a new question as to what really determines the orienting response to begin with. A quick answer would be that memory guides it, and the results of the "Familiarity vs. Novelty" study assist our understanding here (Fig. 5). When one makes an explicit preference decision, that triggers a choice action, which in tern leads to some feedback from the environment (either a reward, or a failure in getting it; either a punishment or a success in avoiding it, etc.). Such an outcome of a choice action will no doubt update a "reward map" in the memory structure. And it is precisely this structure that one would expect to guide orienting responses, albeit implicitly. Indeed, there is evidence in eye-movement literature that a reward system is activated before an eye movement to occur. The results of the "Familiarity vs. Novelty' study suggest only that the way the reward map is updated and how it triggers orienting may be different across object categories. 


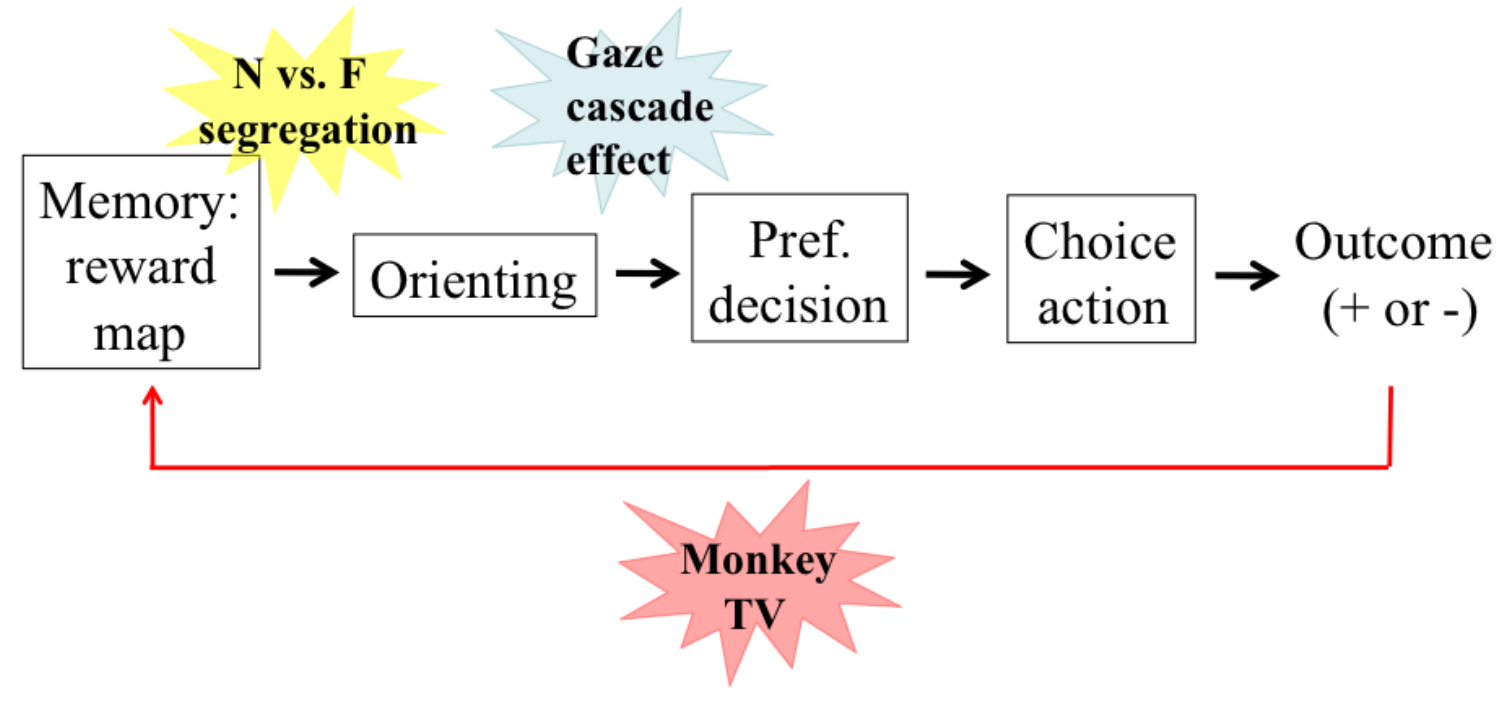

Figure 5. Schematic diagram of preference feedback loop.

This positive-feedback loop may account for, and be consistent with, a variety of observations. Just to name a few; why one needs to make multiple saccades and gazes before consciously determining its choice, why the gaze cascade must occur to make a preference choice in a very wide range of conditions, etc. More importantly, this framework raises a very intriguing possibility, i.e. perhaps seeing is rewarding for its own sake. Indeed, there is a line of anatomical evidence to indirectly support this. There is increasing density of a type of opioid receptor from the primary to the higher-level visual cortices (Lewis, et al. 1981). Also, our own multiple recording data from the OFC (Orbito Frontal Cortex) of alert monkey suggest that there are a subset of reward-related neurons (approximately one quarter) that respond exclusively to the reward-value of watching video, another subset (a quarter) that respond to both video and juice reward (Campos et al., 2008). Thus, seeing itself is rewarding in the veryneuroscientific sense in the primates at least, presumably owing to the positive feedback loop described above.

Taken together, the findings point to the new view of preference (and possibly beauty) decision. According to this view, preference decision making does not occur at a moment in time, even though our phenomenological experience at a conscious level suggest so. Rather, it should be regarded as a more dynamical, somatic and implicit process occurring over short- and long-time scales. 


\section{REFERENCES}

Campos, M., Koppitch, K., Andersen, R. A., \& Shimojo, S. Overlapping representation of juice and video rewards in primate OFC [Abstract]. Journal of Vision, 8(6):546, 546a, http://journalofvision.org/8/6/546/, doi:10.1167/8.6.546 (2008).

Haith, M. M., Bergman, T., \& Moore, M. J. Eye contact and face scanning in early infancy. Science, 198, 853-855(1977).

Kim, H., Adolphs, R., O'Doherty, J. P.,\&Shimojo, S.Temporal isolation of neural processes underlying face preference decision. Proceedings of the National Academy of Sciences, 104,18253-18258(2007).

Lewis, M., Mishkin, M., Bragin, E., Brown, R. M., Pert, C. B., \& Pert, A. Opiate receptor gradientsin monkey cerebral cortex: correspondence with sensory processing hierarchies. Science,Science, 211, 13, 1166-1169 (1981).

Liao, H.-I., \& Shimojo, S. Novelty vs. familiarity principles in preference decision: task-context of memory matters [Abstract]. Journal of Vision, 8(6):522, 522a, http://journalofvision.org/8/6/522/, doi:10.1167/8.6.522(2008).

Simion, C.,\&Shimojo, S.Early interactions between orienting, visual sampling and

decision making in facial preference. Vision Research, 46, 3331-3335(2006a).

Simion, C. and Shimojo, S. A systematic investigation of the gaze manipulation effect. Vision Sciences Society (Abstract), 75 (2006b).

Shimojo, S., Simion, C,\&Changizi, M. Gaze and preference - orienting behavior as a somatic precursor of preference decision. The Science of Social Vision, R. B. Adams, Jr., N. Ambady, K. Nakayama, \& S.Shimojo(Eds.), Oxford University Press, New York (in press).

Shimojo, S., Simion, C., Shimojo, E., and Scheier, C. Gaze bias both reflects and influences preference.Nature Neuroscience, 6, 1317-1322(2003)..

Shimojo, E., Park, J., Lebon, L., Schleim, S., \& Shimojo, S. Familiarity vs. novelty principles for preference [Abstract]. Journal of Vision, 7(9):933, 933a, http://journalofvision.org/7/9/933/, doi:10.1167/7.9.933 (2007).

Shimojo, E., Park, J., Kashino, M., \& Shimojo, S. Familiarity for faces and novelty for natural scenes in preference: Does similarity matter? [Abstract]. Journal of Vision, 8(6):1137, 1137a, http://journalofvision.org/8/6/1137/, doi:10.1167/8.6.1137 (2008).

Zajonc, R. B. Attitudinal effects of mere exposure.Journal of Personality and Social Psychology Monograph Supplement, 9, 2, 2, 1-27 (1968). 